\title{
MOTIVOS DE CONSULTA MAS FRECUENTES EN 620 ADOLESCENTES
}

\section{Estudio prospectivo en el Hospital Universitario San Vicente de Paúl Julio 15 de 1970 a Julio 14 de 1971.}

\author{
Dr. Alfonso Júbiz H.* \\ Dr. Gabriel A. Tobón L.** \\ Sr. Daniel Hoyos***
}

CONTENIDO :

INTRODUCCION.

OBJETIVOS,

UNIVERSO Y MUESTRA.

MATERIAL Y METODOS.

RESULTADOS $Y$ ANALISIS.

CONCLUSIONES.

RESUMEN.

BIBLIOGRAFIA.

\section{I - Introducción}

Ha sido costumbre considerar a la mujer adolescente como un ser aislado, incomprendido, apartado del resto de sus semejantes porque dejó de ser niña y todavía no es adulta, en esta época ocurre la transición de estas edades ( 1 ).

Algunos autores están de acuerdo en afirmar que en los textos de Ginecología se menciona muy poco sobre este grupo de mujeres y lo hacen sólo para referirse al capítulo de malformaciones congénitas (2).

Con los avances de la civilización las adolescentes solicitan y exigen mayor atención, la cual debe ser siempre brindada por un grupo multidis-

ciplinario, no solamente de médicos sino también de educadores sexuales, psicólogos, etc. (3).

Es frecuente observar que las adolescentes presentan problemas relacionados con la maduración psicosexual, falta casi absoluta de instrucción tanto en el hogar como en la escuela. Es raro encontrar adolescentes que hayan recibido una preparación adecuada para la menarca; este hecho, fisiológico, es aceptado como algo bochornoso que causa rechazo en el resto del grupo. Toda esta deficiente preparación a la vida sexual hace de la adolescente una persona inmadura, con una serie de problemas emocionales y sexuales que en muchas ocasiones pueden prevenirse. Estas pacientes llegan a la primera relación sexual ignorantes de sus consecuencias, el resultado es el embarazo no deseado que tantas implicaciones representa a la mujer, al hi-

* Profesor Agregado de Obstetricia y Ginecología, U. de A.

** Residente III de Obstetricia y Ginecología, U. de A.

*** Estadístico Facultad de Medicina, U. de A. 
jo, a la sociedad y a las instituciones de asistencia pública.

Todos los días asistimos en los hospitales al ingreso de adolescentes embarazadas que no conocen nada sobre la función reproductiva. La mayoría de ellas solteras, casi todas vienen de un grupo socio-económico bajo en las cuales hay un núcleo familiar patológico, sin un apoyo emocional adecuado, mal alimentadas, sin control prenatal, generalmente no cumplen las citas ni siguen las prescripciones que se dan para prevenir complicaciones del embarazo, parto o puerperio. Todos estos agravantes aumentan el riesgo obstétrico, frecuente en estas pacientes.

Una consecuencia frecuente del embarazo no deseado es el aborto criminal, el cual representa una complicación grave en obstetricia y un problema social y de salud pública, ampliamente analizado en Colombia y América. (4, 5, 6, 7, 8).

Al emprender el presente estudio consideramos que podemos contribuir al mejor conocimineto de los problemas que se presentan a la mujer en esta etapa de la vida, ya que este grupo constituye un porcentaje alto de nuestra población (9).

\section{II - Objetivos}

1. Analizar los motivos de consulta más frecuentes en las adolescentes que asisten al Departamento de Obstetricia y Ginecología del Hospital Universitario San Vicente de Paúl durante un año (Julio 15 de 1970 - Julio 14 de 1971).

2. Determinar la incidencia del aborto en estas pacientes.

3. Determinar la incidencia del parto, al igual que el método y duración del mismo.
4. Determinar la edad promedio de la menarca y los factores relacionados con ella (instrucción previa, ciclos iniciales, cantidad y dismenorrea).

5. Determinar la edad de la primera relación sexual.

\section{III - Universo y Muestra}

El universo para el presente estudio está constituído por 620 pacientes adolescentes de clase socio-económica inferior que asistieron al Departamento de Obstetricia y Ginecología del Hospital Universitario San Vicente de Paúl durante un año. La muestra es el mismo universo, siendo ésta seleccionada, voluntaria y hospitalaria, representativa de la población antes especificada.

\section{IV - Material y Métodos}

Definimos como adolescente a toda mujer cuya edad esté comprendida entre 10 y 18 años (10).

El presente estudio se efectuó en el Departamento de Obstetricia y Ginecología del Hospital Universitario San Vicente de Paúl en el lapso de tiempo comprendido entre el 15 de Julio de 1970 y el 14 de Julio de 1971.

Se incluyeron todas las pacientes adolescentes que consultaron en el tiempo antes anotado. Se diseñó un formulario (Anexo No 1) el cual fue probado previamente.

La obtención de los datos se hizo por entrevista directa practicada por uno de nosotros. La tabulación y análisis de los resultados se hizo por el sistema IBM. Las variables que se $\mathrm{mi}$ dieron fueron: edad, estado civil, motivo de consulta, edad de la menarca, instrucción previa a la menarca, ciclo inicial, cantidad de la menstruación, dismenorrea, edad de la prime- 
PROTOCOLO DE HISTORIA PARA ESTUDIO DE PROBLEMAS OBSTETRICO Y GINECOLOGICO EN LA ADOLESCENCIA.

DEPARTAMENTO DE OBSTETRICIA Y GINECOLOGIA UNIVERSIDAD DE ANTIOQUIA- HOSPITAL UNIVERSITARIO SAN VICENTE DE PAUL

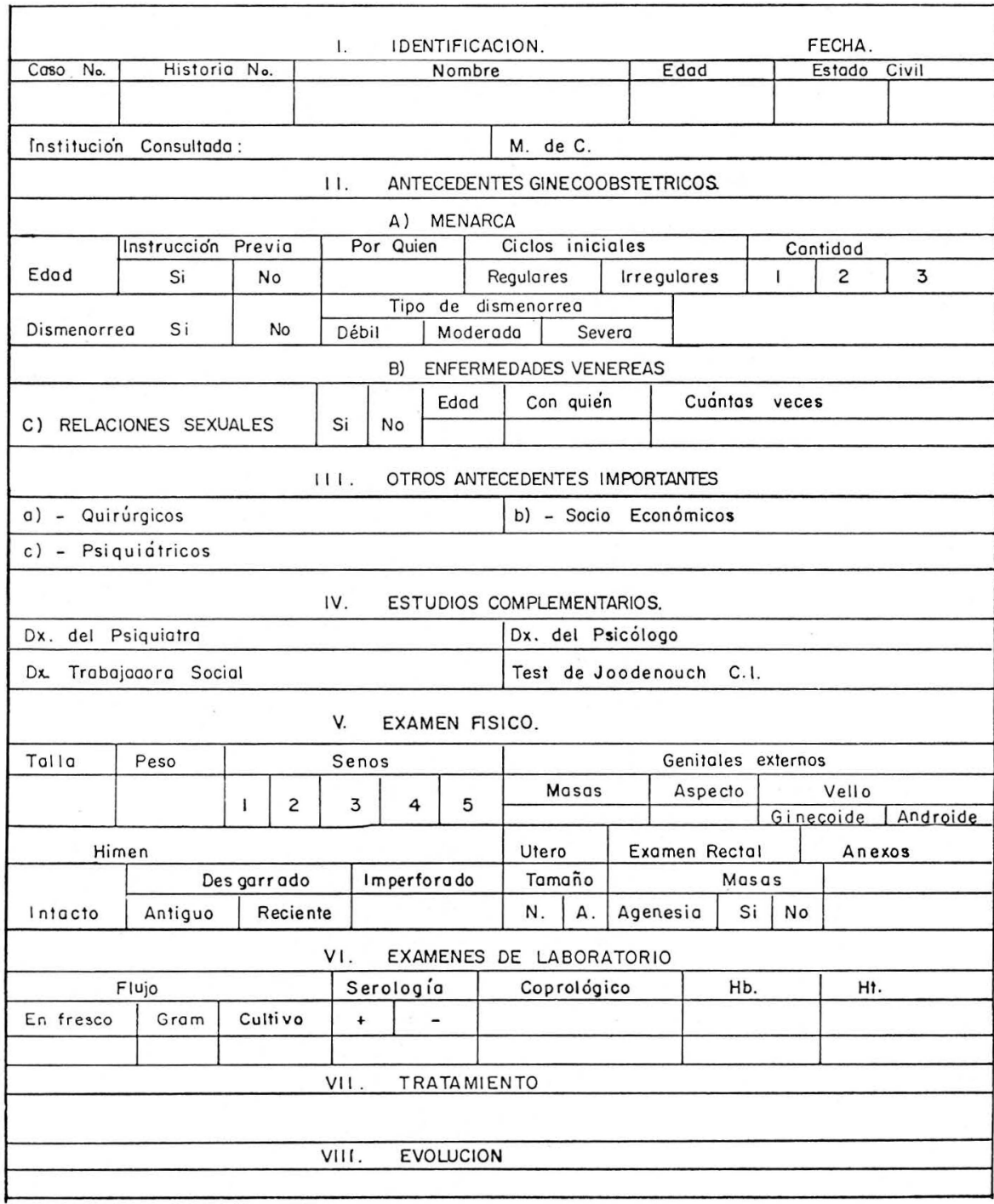


ra relación sexual, número de embarazos, número de abortos, consulta prenatal, semanas de embarazo, método del parto, duración del trabajo de parto en horas, tratamiento quirúrgico, diagnóstico definitivo, talla y peso de la madre, resultado de la serología y hemoglobina, sexo y peso del niño.

Al analizar el ciclo menstrual inicial de cada paciente consideramos que era regular cuando éste se presentaba con una frecuencia de 21 a 35 días. La cantidad fue evaluada por el número de toallas higiénicas utilizadas durante cada día de la hemorragia así: 3 a 4 poca, 5 a 8 regular, más de 8 mucha. La dismenorrea se clasificó en débil, moderada o severa, según la intensidad del dolor, el uso de analgésicos o sedantes y la incapacidad física.

Consideramos que el trabajo de parto era inicial cuando la paciente ingresaba con una dilatación inferior a $5 \mathrm{cms}$. y trabajo de parto avanzado cuando éste era mayor de $5 \mathrm{cms}$. Cuando la paciente consultó por más de dos síntomas se tabuló el más importante para la paciente pero a juicio del médico.

Se buscó la asociación de los motivos de consulta según grupo de edad, el embarazo según la edad y el estado civil, el método del parto según la edad y el estado civil, la menarca con los factores relacionados con ella.

Estamos convencidos que en nuestro estudio faltó el análisis de los factores emocionales y psiquiátricos de cada paciente. No dudamos de la importancia que ellos representan en este grupo de mujeres, no los estudiamos por la carencia de recursos económicos y de personal pero insistimos en que son factores de primer orden en el análisis de los problemas de la adolescente.

\section{V - Resultados y Análisis \\ CUADRO NN 1}

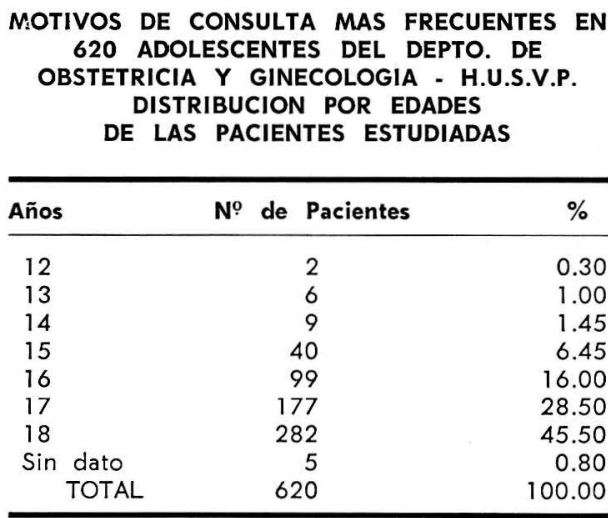

Edad promedio 17 años.

Desviación standard I.

Mediana 17 años.

Cuadro № 1: Distribución por edades de 620 pacientes.

Al analizar el presente Cuadro podemos observar que de las 620 pacientes estudiadas, $57(9,2 \%)$ eran menores de 15 años.

Durante el tiempo del presente estudio ingresaron al Servicio 6.374 pacientes y encontramos que las menores de 15 años, representan el $0,8 \%$. Si este dato lo comparamos con el encontrado en $u$ nestudio anterior efectuado en el mismo Servicio, hace 11 años, se aprecia un aumento diez veces mayor (11).

Este hecho ha sido comprobado en otros países como Inglaterra y Gales donde el aumento de las adolescentes menores de 15 años fue cinco veces mayor en el transcurso de 10 años (12). Clough (13) estableció en $45 \%$ el aumento de primíparas adolescentes de 16 años, estas cifras ha- 
blan de la magnitud de los problemas que este grupo de pacientes representa para las instituciones de asistencia pública y para la sociedad.

Cuadros Nos. 2, 3, 4: Motivos de Consulta y Diagnóstico definitivo.

\section{CUADRO № 2}

\section{MOTIVOS DE CONSULTA MAS FRECUENTES EN \\ 620 ADOLESCENTES DEL DEPTO. DE \\ OBSTETRICIA Y GINECOLOGIA - H.U.S.V.P. \\ JULIO 15-70 - JULIO 14-71 \\ MOTIVOS DE CONSULTA}

\begin{tabular}{lrr}
\hline Motivos & No & $\%$ \\
\hline Trabajo de parto inicial & 291 & 46.9 \\
Trabajo de parto avanzado & 176 & 28.4 \\
Hemorragia & 68 & 11.0 \\
Dolor abdominal & 21 & 3.4 \\
Admisión post-parto & 13 & 2.1 \\
Fiebre & 13 & 2.1 \\
Embarazada no en trabajo & 10 & 1.6 \\
Convulsiones & 12 & 1.9 \\
Otros & 16 & 2.6 \\
TOTAL & 620 & 100.0 \\
\hline & & \\
& & grafica
\end{tabular}

Nuestras pacientes consultaron principalmente por embarazo y metrorragia, el $75 \%$ de ellas ingresaron por trabajo de parto inicial o avanzado y de éstas el $40 \%$ lo hicieron cuando el trabajo de parto estaba avanzado, dato este de mucho interés porque confirma la creencia general de que las embarazadas jóvenes consultan tardíamente durante el parto lo cual dificulta la atención y prevención de las complicaciones: 7 pacientes tenían 14 años. Las pacientes que consultan a este hospital pertenecen a la clase socio-económica pobre, sin instrucción elemental y con un desconocimiento de métodos anticonceptivos (14). Baird (15) señala que las primíparas más jóvenes se encuentran frecuentemente en las clases socio-económicas más pobres.

65 pacientes consultaron por aborto incompleto lo cual representa del total analizado un $10,6 \%$, al buscar Nol

MOTIVOS DE CONSULTA MAS FRECUENTES EN 620

ADOLESCENTES DEL DPTO DE OBSTETRICIA $Y$ GINECOLOGIA

H.U.S.V.P Julio 15/70 - Julio 14/71

MOTIVOS DE CONSULTA

Trabajo de parto inıcial

Trabajo de parto avanzado

Hemorragıa.

Dolor abdominal

Admision post-parto

Fiebre

Convulsiones

Embarazada no en trabajo Otros motivos

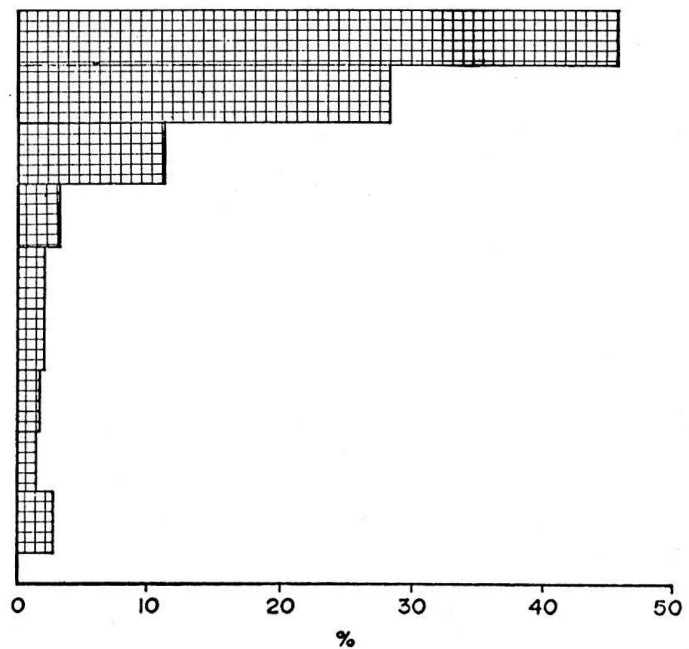


cuadro $\mathrm{N}^{03}$

MOTIVOS DE CONSULTA MAS FRECUENTES EN 620 ADOLESCENTES DEL DPTO DE OBSTETRICIA Y GINECOLOGIA H.U.S.V.P Julio 15/70 - Julio 14/71 MOTIVOS DE CONSULTA SEGUN GRUPOS DE EDAD

\begin{tabular}{|c|c|c|c|c|c|c|c|c|c|c|c|c|}
\hline \multirow{3}{*}{ MOTIVO DE CONSULTA } & \multicolumn{10}{|c|}{ EDADES } & \multirow{2}{*}{\multicolumn{2}{|c|}{ TOTAL }} \\
\hline & \multicolumn{2}{|c|}{$14^{x}$} & \multicolumn{2}{|c|}{15} & \multicolumn{2}{|c|}{16} & \multicolumn{2}{|c|}{17} & \multicolumn{2}{|c|}{18} & & \\
\hline & $\mathrm{N}^{2}$ & $\%$ & $\mathrm{~N}^{\circ}$ & $\%$ & Na & $\%$ & $\mathrm{~N}^{\circ}$ & $\%$ & $\mathrm{~N}^{2}$ & $\%$ & $\mathrm{~N}^{2}$ & $\%$ \\
\hline TRABAJO DE PARTO INICIAL & 3 & - & 19 & 47.5 & 51 & 51.5 & 79 & 44.6 & 137 & 48.6 & 289 & 47.7 \\
\hline TRABAJO DE PARTO AVANZADO & 4 & - & 9 & 22.5 & 20 & 20.3 & 60 & 33.9 & 80 & 28.3 & 173 & 28.7 \\
\hline HEMORRAGIA & 1 & - & 6 & 15.0 & 9 & 9.1 & 16 & 9.0 & 31 & 11.0 & 63 & 10.6 \\
\hline .DOLOR ABDOMINAL & 1 & - & 1 & 2.5 & 6 & 6.1 & 2 & 1.2 & 11 & 3.9 & 21 & 3.4 \\
\hline FIEBRE & - & - & 3 & 7.5 & 3 & 3.0 & 3 & 1.7 & 4 & 1.4 & 13 & 2.1 \\
\hline ADMISION POST-PARTO & - & - & - & - & 4 & 4.0 & 4 & 2.2 & 5 & 1.8 & 13 & 2.1 \\
\hline CONVULSIONES & - & - & 2 & 5.0 & 3 & 3.0 & 2 & 1.2 & 5 & 1.8 & 12 & 1.9 \\
\hline EMBARAZADA NO EN TRABAJO & - & - & - & - . & 2 & 2.0 & 3 & 1.7 & 5 & 1.8 & 10 & 1.6 \\
\hline OTROS & - & - & - & - & 1 & 1.0 & 8 & 4.5 & 4 & 1.4 & 13 & 2.1 \\
\hline TOTAL & 9 & - & 40 & 100.0 & 99 & 100.0 & 177 & 100.0 & 282 & 1000 & 607 & 1000 \\
\hline
\end{tabular}

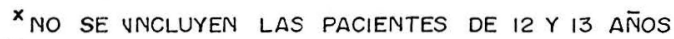

${ }^{x}$ HAY 5 PACIENTES SIN DATO.

grafica $\mathrm{N}^{\circ} 2$

MOTIVOS DE CONSULTA MAS FRECUENTES EN 620 ADOLESCENTES DEL DPTO DE OBSTETRICIA Y GINECOLOGIA H.U.S.V.P Julio $15 / 70$ - Julio $14 / 71$ MOTIVOS DE CONSULTA SEGUN EDAD

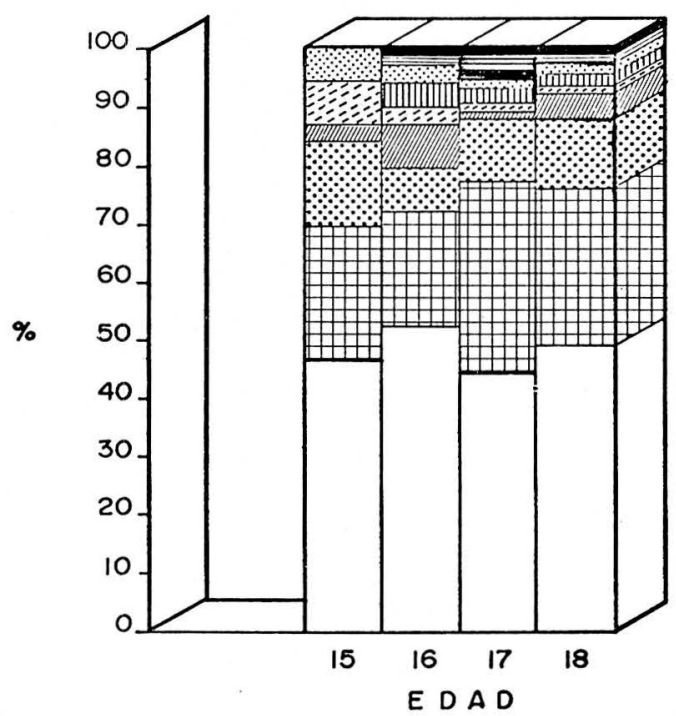

\begin{tabular}{|c|c|}
\hline & TRAB. PARTO INICIAL \\
\hline 世正 & TRAB. PARTO AVANZADO \\
\hline 6 & HEMORRAGIA \\
\hline & DOLOR ABDOMINAL \\
\hline 非㳔 & FIEBRE \\
\hline 而血 & ADMIS. POST-PARTO \\
\hline 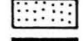 & CONVULSIONES \\
\hline 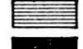 & EMBARAZADA NO EN TRAB. \\
\hline
\end{tabular}

$I=$ FALTAN LAS EDADES 12,13 Y 14 POR EL POCO N DE PACIENTES 
el grado de infección nos llamó la atención que $32(49 \%)$ de ellas te. nían un aborto séptico III. Esta cifra es dos veces y media más, que la encontrada en un estudio reciente efectuado en el mismo Servicio (8).

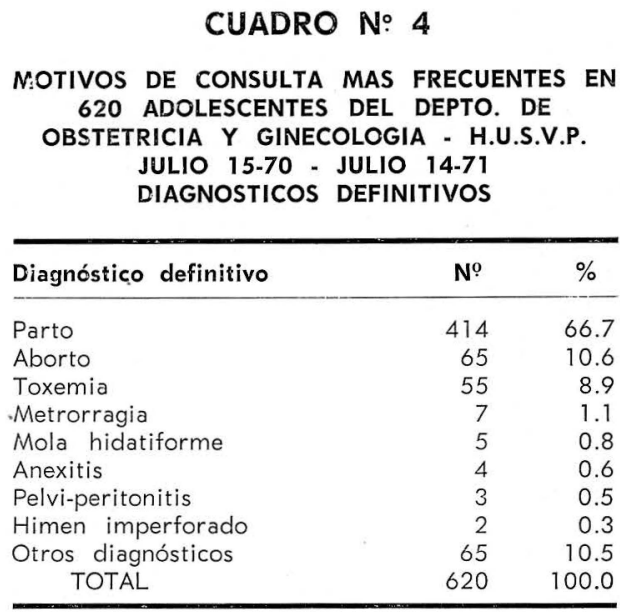

La eclampsia fue cinco veces más frecuente en las adolescentes que en las mayores de 20 años (16). Algunos autores afirman que la eclampsia ocurre tres a cinco veces más en pacientes jóvenes que en pacientes de cdad superior a los 20 años (17).

Nokes (18) encontró $18 \%$ de incidencia de preeclampsia en adolescentes. Clark (19) en un estudio comparativo encontró que a pesar de los cuidados prenatales el $11 \%$ de las adolescentes desarrollaron toxemia. Las dietas anormales y el exceso de peso son dos de los más importantes problemas en el manejo de adolescentes embarazadas. Se considera que hay otros factores propios de estas jóvenes que influyen para que desarrollen la enfermedad. De nuestras pacientes solo el 23,2\% había asistido a la Consulta Prenatal.

Cuadro No: 5: Embarazo según la edad y el estado civil.

grafica $N^{\circ} 3$

MOTIVOS DE CONSULTA MAS FRECUENTES EN 620 ADOLESCENTES DEL DPTO DE OBSTETRICIA Y GINECOLOGIA

H.U.S.V.P Julio $15 / 70$ - Julio $14 / 71$ DIAGNOSTICOS

Parto

Aborto

Toxemıa

Metrorragıa

Mola hıdatiforme

Anexitis

Pelvi-peritonıtis

Hımen imperforado

Otros diagnosticos

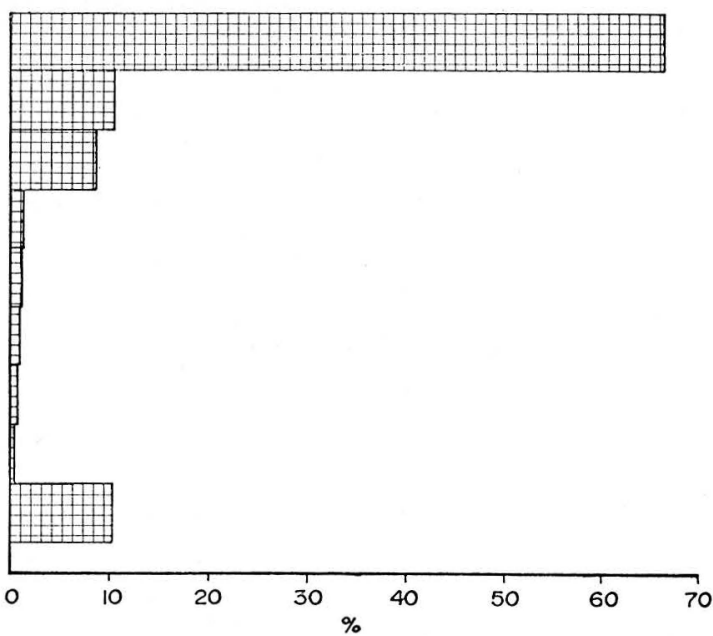


cuadro $\mathrm{N}^{\circ} 5$

MOTIVOS DE CONSULTA MAS FRECUENTES EN 620 ADOLESCENTES DEL DPTO DE OBSTETRICIA $Y$ GINECOLOGIA H.U.S.V.P Julio $15 / 70$ - Julio $14 / 71$ EMBARAZOS SEGUN EDAD Y ESTADO CIVIL

\begin{tabular}{|c|c|c|c|c|c|c|c|c|c|}
\hline \multirow{2}{*}{ EDADES } & \multirow{2}{*}{$\begin{array}{c}N^{\circ} \\
\text { EMBARAZ. }\end{array}$} & \multicolumn{2}{|c|}{ SOLTERAS } & \multicolumn{2}{|c|}{ CASADAS } & \multicolumn{2}{|c|}{ VIUDAS } & \multicolumn{2}{|c|}{ TOTAL } \\
\hline & & $\mathrm{N}^{\circ}$ & $\%$ & $\mathrm{~N}^{2}$ & $\%$ & $\mathrm{~N}^{\circ}$ & $\%$ & $\mathrm{~N}^{\circ}$ & $\%$ \\
\hline 13 & 1 & 1 & - & 1 & - & - & - & 2 & - \\
\hline 14 & 1 & 6 & - & 1 & - & - & - & 7 & - \\
\hline 15 & $\begin{array}{l}1 \\
2\end{array}$ & $\begin{array}{r}28 \\
1\end{array}$ & $\begin{array}{r}96.5 \\
3.5\end{array}$ & $\begin{array}{l}8 \\
1\end{array}$ & - & - & $\overline{-}$ & $\begin{array}{r}36 \\
2\end{array}$ & $\begin{array}{r}94.7 \\
5.3\end{array}$ \\
\hline 16 & $\begin{array}{l}1 \\
2\end{array}$ & $\begin{array}{r}57 \\
4\end{array}$ & $\begin{array}{r}93.4 \\
6.6 \\
\end{array}$ & $\begin{array}{r}31 \\
5\end{array}$ & $\begin{array}{l}86.1 \\
13.9 \\
\end{array}$ & - & $\begin{array}{l}- \\
-\end{array}$ & $\begin{array}{r}88 \\
9 \\
\end{array}$ & $\begin{array}{r}90.6 \\
9.4 \\
\end{array}$ \\
\hline 17 & $\begin{array}{l}1 \\
2 \\
3 \\
4\end{array}$ & $\begin{array}{c}82 \\
13 \\
- \\
-\end{array}$ & $\begin{array}{c}86.3 \\
13.7 \\
- \\
-\end{array}$ & $\begin{array}{r}47 \\
26 \\
2 \\
1 \\
\end{array}$ & $\begin{array}{r}61.8 \\
34.2 \\
2.6 \\
1.3\end{array}$ & $\begin{array}{l}- \\
- \\
-\end{array}$ & $\begin{array}{l}- \\
- \\
- \\
-\end{array}$ & $\begin{array}{r}129 \\
39 \\
2 \\
1\end{array}$ & $\begin{array}{r}75.4 \\
22.8 \\
1.2 \\
0.6\end{array}$ \\
\hline 18 & $\begin{array}{l}1 \\
2 \\
3 \\
4 \\
5 \\
\end{array}$ & $\begin{array}{r}112 \\
26 \\
3 \\
- \\
1\end{array}$ & $\begin{array}{r}78.9 \\
18.3 \\
2.1 \\
- \\
0.7 \\
\end{array}$ & $\begin{array}{r}84 \\
38 \\
11 \\
1 \\
1\end{array}$ & $\begin{array}{r}62.2 \\
28.2 \\
8.2 \\
0.7 \\
0.7 \\
\end{array}$ & $\begin{array}{l}1 \\
- \\
- \\
-\end{array}$ & $\begin{array}{l}- \\
- \\
- \\
-\end{array}$ & $\begin{array}{r}197 \\
64 \\
14 \\
1 \\
1 \\
\end{array}$ & $\begin{array}{r}71.1 \\
23.1 \\
5.0 \\
0.4 \\
0.4 \\
\end{array}$ \\
\hline SIN DATO & $\begin{array}{l}1 \\
2 \\
\end{array}$ & $\begin{array}{l}2 \\
2 \\
\end{array}$ & - & $i$ & 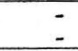 & - & - & $\begin{array}{l}2 \\
3\end{array}$ & - \\
\hline TOTAL & $\begin{array}{l}1 \\
2 \\
3 \\
4 \\
5\end{array}$ & $\begin{array}{r}288 \\
46 \\
3 \\
- \\
1 \\
338 \\
\end{array}$ & $\begin{array}{r}85.2 \\
13.6 \\
0.9 \\
- \\
0.3 \\
100.0 \\
\end{array}$ & $\begin{array}{r}172 \\
71 \\
13 \\
2 \\
1 \\
259 \\
\end{array}$ & $\begin{array}{r}66.6 \\
27.4 \\
5.0 \\
0 . .8 \\
0.2 \\
100.0\end{array}$ & $\begin{array}{l}1 \\
- \\
- \\
- \\
1\end{array}$ & $\begin{array}{l}- \\
- \\
- \\
- \\
-\end{array}$ & $\begin{array}{r}461 \\
117 \\
16 \\
2 \\
2 \\
598 \\
\end{array}$ & $\begin{array}{r}77.1 \\
19.6 \\
2.7 \\
0.3 \\
0.3 \\
100.0 \\
\end{array}$ \\
\hline
\end{tabular}

\section{CUADRO N: 6}

MOTIVOS DE CONSULTA MAS FRECUENTES EN 620 ADOLESCENTES DEL DEPARTAMENTO DE OBSTETRICIA Y GINECOLOGIA - H.U.S.V.P. — JULIO 15.70 - JULIO 14.71 METODO DEL PARTO SEGUN EL ESTADO CIVIL

\begin{tabular}{|c|c|c|c|c|c|c|c|c|}
\hline & \multicolumn{2}{|c|}{ Solteras } & \multicolumn{2}{|c|}{ Casadas } & \multicolumn{2}{|c|}{ Viudas } & \multicolumn{2}{|c|}{ Total } \\
\hline & $\mathrm{N}^{0}$ & $\%$ & $\mathbf{N}^{0}$ & $\%$ & $\mathbf{N}^{o}$ & $\%$ & $\mathbf{N}^{o}$ & $\%$ \\
\hline Espontáneo & 166 & 64,5 & 322 & 67,1 & - & - & 156 & 70,3 \\
\hline Fórceps & 66 & 25,7 & 109 & 22,7 & - & - & 43 & 19,5 \\
\hline Cesárea & 14 & 5,4 & 26 & 5,4 & - & - & 12 & 5,4 \\
\hline Ext. parcial & 8 & 3,2 & 13 & 2,7 & - & - & 5 & 2,2 \\
\hline Vacuum & 1 & 0,4 & 6 & 1,3 & - & - & 5 & 2,2 \\
\hline Ext. total & 1 & 0,4 & - & 0,4 & 1 & - & - & 12 \\
\hline Otros & 1 & 0,4 & 2 & 0,4 & - & - & 1 & 0,4 \\
\hline TOTAL & 257 & 100,0 & 480 & 100,0 & 1 & - & 222 & 100,0 \\
\hline
\end{tabular}

De las 620 pacientes estudiadas, $598(96,4 \%)$ tenían uno o más embarazos. Llama la atención que a los 16 años el $10 \%$ tenían dos embarazos, pero a los 18 este porcentaje se eleva a $23 \%$. Observamos también que a los 17 años una paciente tenía
4 embarazos y a los 18, otra había tenido 5 embarazos. Las relaciones sexuales prematrimoniales y la falta absoluta de conocimientos y métodos anticonceptivos hace que esta cifra sea cada vez mayor. En relación al estado civil el $56,5 \%$ eran solteras, esto 
en realidad no influye para el número de embarazos.

Cuadros Nos. 6 y 7: Método del parto según la edad y el estado civil.

No encontramos diferencia del estado civil en relación al método del parto, solo que el fórceps es un poco más frecuente en las solteras $25,7 \%$.

$\mathrm{Al}$ analizar las edades, observamos que el parto espontáneo es igualmente frecuente en todos los grupos $(66,9 \%)$.

En $108(22,7 \%)$ de las 475 pacientes atendidas para el parto se utilizó en él, el fórceps. La cesárea en el grupo de adolescentes es de $5,6 \%$, prácticamente igual al dato encontrado al revisar hace un año los archivos del Departamento, que fue de $5,2 \%$.
Cuadro № 8: Relación entre el peso del niño y las semanas de gestación.

La edad promedio de la gestación fue de 39 semanas, dato que consideramos normal en nuestro medio.

El $55 \%$ de los niños tenían un peso que fluctuó entre 2.001 a 3.000 gms. El $43 \%$ de los niños pesaron menos de $2.500 \mathrm{gms}$. Las adolescentes tienen niños de bajo peso según algunos autores (12). Otros consideran que la prematuridad es esencialmente la misma en el niño de la adolescente, que en el de la madre adulta dentro de un mismo nivel socio-económico.

\section{Tratamiento quirúrgico}

113 pacientes fueron sometidas a tratamiento quirúrgico mayor o me-

cuadro $N^{2} 7$

MOTIVOS DE CONSULTA MAS FRECUENTES EN 620

ADOLESCENTES DEL DPTO DE OBSTETRICIA Y GINECOLOGIA

H.U.S.V.P Julio $15 / 70$ - Julio 14/71

METODO DEL PARTO SEGUN LA EDAD

\begin{tabular}{|c|c|c|c|c|c|c|c|c|c|c|c|c|c|c|c|}
\hline \multirow{2}{*}{ EDADES } & \multicolumn{2}{|c|}{ ESPONTAN. } & \multicolumn{2}{|c|}{ FORCEPS } & \multicolumn{2}{|c|}{ CESAREA } & \multicolumn{2}{|c|}{ EX.PARCIAL } & \multicolumn{2}{|c|}{ VACUUM } & \multicolumn{2}{|c|}{ EX.TOTAL } & \multicolumn{2}{|c|}{ OTROS } & \multirow{2}{*}{ TOTAL } \\
\hline & $\mathrm{N}^{\circ}$ & $\%$ & $\mathrm{~N}^{\circ}$ & $\%$ & $\mathrm{~N}^{\circ}$ & $\%$ & $\mathrm{~N}^{\circ}$ & $\%$ & $\mathrm{~N}^{\circ}$ & $\%$ & $\mathrm{~N}^{\circ}$ & $\%$ & $\mathrm{~N}^{\circ}$ & $\%$ & \\
\hline 14 AÑNOS & 6 & - & 1 & - & - & - & - & - & - & - & - & - & - & - & 7 \\
\hline $15 "$ & 19 & 65.5 & 8 & 27.6 & 2 & 6.9 & - & - & - & - & - & - & - & - & 29 \\
\hline $16 "$ & 51 & 69.8 & 11 & 15.0 & 9 & 12.5 & 2 & 2.7 & - & - & - & - & - & - & 73 \\
\hline $17 \quad$ & 99 & 67.8 & 28 & 19.2 & 8 & 5.5 & 6 & 4.1 & 3 & 2.0 & - & - & 2 & 1.4 & 146 \\
\hline $18 "$ & 143 & 65.0 & 60 & 27.3 & 7 & 32 & 5 & 2.2 & 3 & 1.4 & 2 & Q9 & - & - & 220 \\
\hline TOTAL & 318 & 66.9 & 108 & 22.7 & 26 & 5.6 & 13 & 2.7 & 6 & 1.3 & 2 & 0.4 & 2 & 0.4 & $475^{*}$ \\
\hline
\end{tabular}

* 5 pacientes no figuraban con la edad. 
nor. En el $64,6 \%$ o sea 73 pacientes se practicó vaciamiento digital o instrumental del útero. De éstos, 65 fueron por aborto y el resto por otra causa de metrorragia.

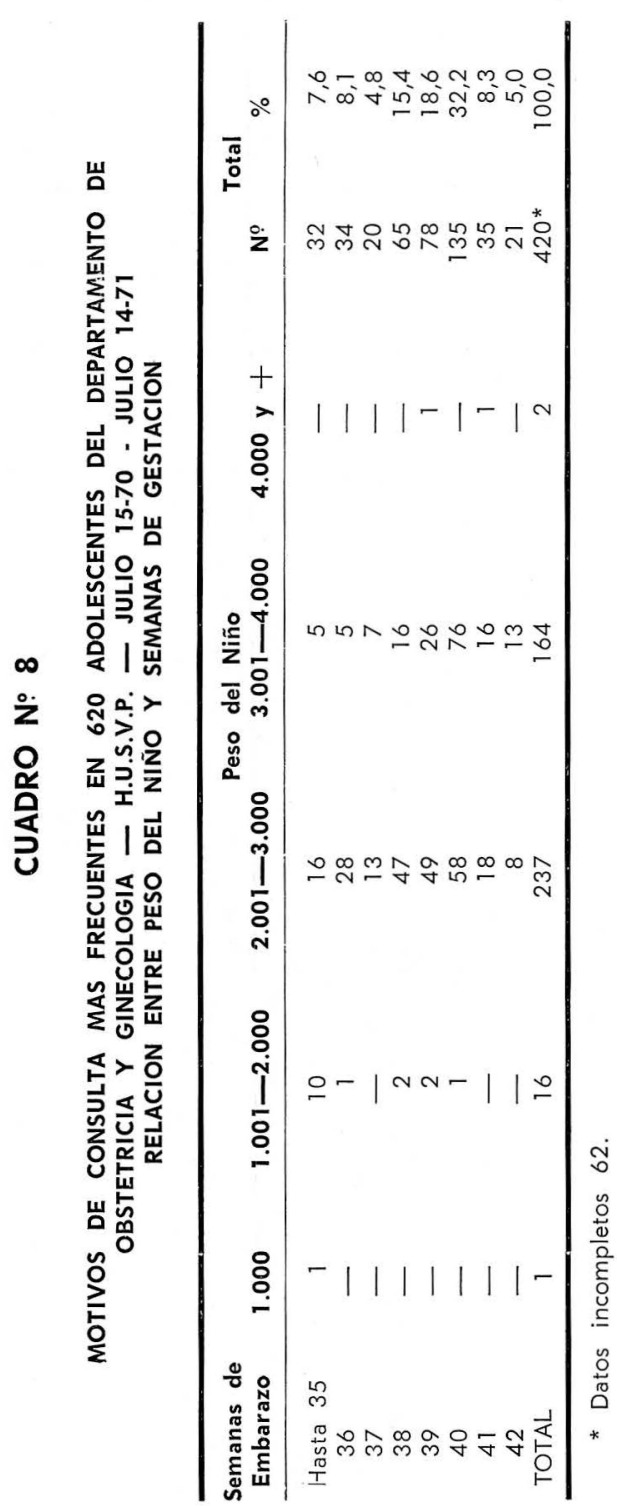

La cesárea ocupó el segundo lugar en el tratamiento quirúrgico con un $23 \%$ las causas más frecuentes fueron posiciones anormales del feto, desproporción cefalopélvica, infección amniótica y sufrimiento fetal.

Vale la pena anotar que a dos pacientes se les sometió a histerectomía abdominal, una por aborto séptico y otra por tromboflebitis pélvica con ligadura de cava por embolia pulmonar.

\section{Menarca}

La edad promedio de la menarca en este grupo de adolescentes fue de 13 años, y el 95\% la tuvieron entre 11 y 15 años. Estos datos están de acuerdo con varios autores $(1,3,20$, 21 ).

Respecto a la instrucción previa sobre la menstruación un 54,7\% dijeron que la habían recibido, pero la fuente principal de esta instrucción fue la madre, la escuela y amigas, creemos que es muy deficiente la instrucción dada por dichas personas por su falta de educación y su nivel cultural.

Otros parámetros averiguados respecto a la menstruación fue su cantidad y encontramos en $52,2 \%$ poca. $41 \%$ de las adolescentes tuvieron dismenorrea siendo débil en $51,5 \%$. Es de anotar que los ciclos iniciales fueron regulares en el 55,3\% de los casos. Datos estos similares encontrados en un estudio hecho anteriormente en este servicio (22).

\section{Relaciones sexuales}

La edad promedio de la primera relación sexual fue a los 15 años, si tenemos en cuenta que la mayoría son pacientes solteras y de las casadas muchas han tenido sus relaciones sexuales prematrimoniales creemos que 
este factor podría jugar papel importante en la tasa de natalidad.

\section{Serología}

La serología positiva en nuestro estudio fue el doble $(4,1 \%)$ a los resultados encontrados en la encuesta nacional de morbilidad. Creemos que los factores analizados anteriormente (promiscuidad sexual, etc.) está influyendo para esta cifra este aumento. Este dato no lo podemos comparar con el grupo general de las pacientes que asisten a este centro asistencial.

\section{Hemoglobina}

De 546 realizadas, el $28,9 \%$ fueron inferiores a 10 gms., el promedio de hemoglobina en todas las pacientes, fue de 10,7 gms., Mussio encontró en 1968 en pacientes menores de 14 años una hemoglobina de menos de 10 gms. en un $24 \%$. (23).

\section{Conclusiones}

1). La edad promedio de las pacientes adolescentes que consultan al Hospital Universitario San Vicente de Paúl es de 17 años.

2). La incidencia del aborto fue de $10,6 \%$.

$3)$. El $75 \%$ de las pacientes ingresaron en trabajo de parto inicial o avanzado.

4). La edad promedio de la menarca fue de 13 años.

5). La edad promedio de la primera relación sexual fue a los 15 años.

6). El $43 \%$ de los niños pesaron menos de 2.500 gms.

7). La mortalidad materna fue de $1,6 \% \times 1.000$.

\section{Resumen}

Se estudian 620 adolescentes en el Departamento de Obstetricia y Ginecología del Hospital Universitario San Vicente de Paúl durante un año (Julio 15 de 1970 a Julio 14 de 1971).

El principal motivo de consulta de estas pacientes fue el parto (75\%). El 10,6\% consultan por aborto. La mortalidad materna fue de $1,6 \times$ 1.000 .

\section{Summary}

Six hundred and twenty adolescents are studied in the obstetrics and gynecological Department of San Vicente de Paul University Hospital during one year (July 15, 1970 to July 14, 1971).

Parturition (birth) was the main reason for consultation of these patients $(75 \%)$.

$10.6 \%$ consulted for abortion. Maternal mortality was $1.6 \times 1.000$.

\section{BIBLIOGRAFIA}

1 DALY, M. J. Desarrollo físico y psicológico de la adolescente. Cl. Obst. y Ginec. Sept. $711,1966$.

2 ALVAREZ, R. R. Simposio sobre la ginecología de la adolescente. Cl. Obst. y Ginec. Sept. 710, 1966.

3 WIDHOLM, O. y col. Gynecological finding in adolescence a study of 514 patients. Acta Obst. \& Gynec. Scandinav. 46: 3, 1967.

4 ARMIJO, R. y MONREAL, T. Epidemiología del aborto provocado en Santiago. Rev. Med. de Chile. 92: 548, 1964.

5 REGENA, M. Condiciones determinantes del aborto inducido. Rev. Med. de Chile. 94: $714,1966$.

6 NEME, B. y col. Obituario materno no abortamento criminoso. An. Brasileros Ginec. 57: 7. 1965 .

7 SANTAMARIA PAEZ, L. E. Aborto séptico y maniobras abortivas. Rev. Col. Obst. y Ginec. $17: 273,1966$. 
8 JUBIZ, A. y col. Estudio sobre aborto séptico. Rev. Col. Obst. y Ginec. 22: 237, 1971.

9 Estudio de Recursos Humanos para la salud y educación médica en Colombia. Minsalud. Ascofame. 1968

10 ZATUCHNI, G. Examen de la adolescente. Cl. Obs. y Ginec. Sep. 722, 1966.

11 GAVIRIA, B. y col. Letalidad perinatal. Rev. Col. Obs. y Ginec. XI May, Jun. 339-350. 1960.

12 RUSSELL, J. K. Pregnancy in the young teen agers. Lancet. II: 365, 1969.

$13 \mathrm{CLOUGH}, \mathrm{W}$. The young primipara Obst. \& Ginec. 12: 373, 1958.

14 JARAMILLO, M. Encuesta de Fecundidad en Medellín. Asoc. Col. Fac. de Medicina. 19. 41. 1968.

15 BAIRD, D. Age and fecundity. British M. J. 2: 1499, 1955.

16 BOTERO, J. TUBERQUIA, J. C. HUERTA, M. Embarazo en mujeres menores de 20 años, comparado con el de otras edades. 19091971. Sin publicar.

17 HUFFMAN. J. The gynecology of chidhood and adolescence. 1a. ed. Philadelphia Saunders, 541, 1968

18 NOKES, J. y col. A critical analysis of the young and elderly primiparas. South. M. J. 45: 866, 1952.

19 CLARK, J. y col. The pregnant adolescent. ann. N. Y. Acad., Sc. 142: 813, 1967.

20 MICHELSEN, J. Estudio epidemiológico y detección precoz del $\mathrm{Ca}$. en las reclusas de la cárcel Nal. de mujeres de Bogotá, D. E. Col. Rev. Col. Obst. y Ginec. Vol. 5; 341. Sept.-Oct. 1969.

21 PECKHAM, C. The relationship between pregnancy weight and certain obstetrics factor. Am. J. Obst. and Gynec. Vol. III (1): I. Sept. 1971

22 MEJIA, W. Estudio sobre aborto y fertilidad en Medellín. Escuela de Salud Pública de U. de A., 1964. ( $\sin$ publicar).

23 MUSsIO, T. Primigrávidas under age 14. Am. J. Obst. Gynec. 84: 442, 1962. 